(c) American Dairy Science Association, 2003.

\title{
Laterality in Bovine Behavior in an Extensive Partially Suckled Herd and an Intensive Dairy Herd
}

\author{
C. J. C. Phillips, S. Llewellyn, and A. Claudia \\ Department of Clinical Veterinary Medicine, \\ University of Cambridge, Cambridge CB3 OES, United Kingdom
}

\begin{abstract}
Cattle exhibit behavioral laterality, but the consistency and correlation between behaviors are unknown. Behavioral laterality was recorded in two herds of contrasting management intensity. The first was a small, extensively managed herd in Brazil, with cows and calves on rangeland, except when removed for handmilking in stalls. The second was a large, intensive British herd, with cows fed mostly indoors and calves removed for individual rearing soon after birth. In herd 1 , the side of the body on which the following behaviors were performed was recorded: rumination (rumination), tail waving (tail), tongue protrusion during the initiation of a feeding bout (feeding), hind leg placement when lying (lying), and front leg initiating walking (walking). The distribution of left and right side dominance was normal for all behaviors, with positive correlations between walking and rumination, tail, and feeding, and between lying and rumination. In herd 2 , rumination, feeding, and lying behaviors were similarly recorded, as well as parlor side-preference (parlor) and the side of a track chosen when returning to pasture (track). For all behaviors except track, the extent of leftand right-side dominance was not normally distributed, and more cows than expected showed strong laterality on the right or the left side. Parlor and track lateralities were correlated, indicating that cows that entered one side of the parlor also tended to choose the same side of the track. Strong laterality in the intensively managed herd therefore contrasted with that observed in the extensively managed herd and the reasons for such differences in laterality are uncertain.
\end{abstract}

(Key words: laterality, asymmetry, handedness, management)

\section{INTRODUCTION}

In animals showing a degree of bilateral symmetry, behaviors involving one of two opposing limbs (e.g., ini-

Received October 15, 2002

Accepted April 2, 2003.

Corresponding author: C. J. C. Phillips; e-mail: cjcp2@cam.ac.uk. tiation of walking) may be performed on either the right or left side of the body, demonstrating a difference in preference or ability between the two sides (Annett, 1985). Such laterality may also be expressed by parts of the body extending sideways during routine behaviors, e.g., tongue and jaw movement when eating. Such behavioral lateralities may be related to asymmetry in body morphology. For example, symmetry has been observed in the diagonal rather than opposing limbs in the four hooves of bovines, which may derive from asymmetrical walking or lying patterns (Phillips et al., 1996).

Behavioral laterality in cattle has also been demonstrated for side preferences in a T-maze (Arave et al., 1992), for entry to a milking parlor (Hopster et al., 1998; Paranhos da Costa and Broom, 2001) and for lying (Uhrbrook, 1969; Arave and Walters, 1980). In relation to lying, it has been observed that heavily pregnant cows show left side laterality (Arave and Walters, 1980; Bao and Giller, 1991), probably because the fetus is positioned towards the right side of the body. Such left side laterality diminishes with age and was not observed in calves (Wilson et al., 1999), suggesting that it is caused by the presence of the fetus in young cows. Albright and Arave (1997) observed that rumination is more common when cows are recumbent on the left side rather than the right side and conclude that left side laterality facilitates rumination. This factor may also increase left side laterality in heavily pregnant, young cows.

In other mammals, laterality in behavior and asymmetry in morphological characteristics at least partly derive from stress that causes cognitive brain lateralization and disruption of cognitive function (Tang and Verstyned, 2002). This influence of stress (a substantial response to noxious or potentially noxious stimuli that carries a biological cost to the animal, Ewbank, 1992) on cognition has been demonstrated in both juvenile (Westergaard et al., 2001) and adolescent (Westergaard et al., 2000) macaques. Developmental instability (physical anomalies and fluctuating anatomic asymmetries) has also been related to the evolution of extreme left- or right-handedness in humans (Gangestad and Yeo, 1994). Neonatal stress is particularly influential in increasing the strength of handedness in both 
rats (Rodriguez-Diaz and Afonso, 1993) and chimpanzees (Bard et al., 1990), but such influences can be attenuated by other behavioral factors later in life (Rodriguez-Diaz and Afonso, 1993). A rat model has demonstrated that extreme handedness diminishes with age (Rodriguez-Diaz and Afonso, 1993), which agrees with the observation of diminished lying side preferences in older cattle by Arave and Walters (1980). In humans, stress-induced lateral tendencies can be alleviated by reducing the stress (Davis, 1988), illustrating the dynamic nature of the phenomenon.

Other factors that are believed to contribute to laterality tendencies in mammals include genetic inheritance, although Arave et al. (1992) found little evidence of this in calf movements to the left or right hand side of a maze. However, it is implicated in the role of development instability on human handedness (Gangestad and Yeo, 1994).

Few studies addressing bovine laterality have investigated whether there are correlations between side preferences in different behaviors. The objective of this observational study was to investigate correlations in laterality between routine behaviors of lactating dairy cows in two herds with contrasting management styles.

\section{MATERIALS AND METHODS}

\section{Experiment 1. Laterality in an Extensive Herd of Restricted Suckled Cows}

This study was conducted using a herd of 40 lactating and 23 nonlactating Holstein-Friesian cows at Fazenda Campolina, Brazil, in spring 2002. The farm had a simple management system based on extensive grazing of the cows with their calves, at an overall stocking rate of one cow + calf per 12 ha. Calves were weaned at 6 mo of age, and the mean saleable milk yield of the cows was approximately $2500 \mathrm{~L} / \mathrm{yr}$. There were also 37 replacement heifers on the farm, which were extensively pastured and received a sugar cane supplement. Thirteen 1-d paddocks of 50 ha each were used in a rotational grazing system; 10 paddocks of Tanzania grass (Panicum maximum cv. Tanzania) and three of Napier grass (Pennisetum purpureum), all containing salt licks and shade. No food supplements were provided except during the winter dry season, when a total of $75 \mathrm{~kg} / \mathrm{cow}$ of concentrate and ad libitum chopped sugar cane (Saccharum spp.) was provided indoors after afternoon milkings over the entire season. Natural service was by two bulls. Cows were hand milked in stalls with their calves tied to their right hand sides at 4 a.m. and 4 p.m., and the cows were showered to relieve heat stress before each milking.

Using only the 40 lactating cows for laterality observations, a total of 11 recordings per cow were taken of which side of the body the following behaviors occurred: feeding (tongue protrusion from one side of the mouth during the initiation of a grazing bout), rumination (dominant side of the mouth occupied by the lower jaw during ruminating), walking (which front leg they moved first after standing still), lying (which hind leg was under the body during lying), and tail (which side of the body the tail was initially moved to during tail swishing). The recordings were made by a single observer close to the herd when they were inside for their supplement (except when recording grazing), during January to March.

Statistical analysis. Behaviors were awarded a score of 0 if the cow exhibited the behavior on the left side and 1 if the cow exhibited the behavior on the right side. The mean score for each cow was obtained for each behavior. All behavior variables were tested for normality of data distribution by the Kolmogorov-Smirnov and Anderson-Darling tests using the statistical package, Minitab (Minitab, 1995). They were normally distributed by the Kolmogorov-Smirnov test, but one of the variables, feeding, was not normally distributed by the Anderson-Darling test. Therefore the mean variables were ranked to determine correlation coefficients by Spearman rank correlations.

\section{Experiment 2. Laterality in an Intensive Dairy Herd}

This study was conducted using a herd of 180 Holstein-Friesian dairy cows at the Cambridge University Farm in the United Kingdom. Calves were removed from their mothers at $1 \mathrm{~d}$ of age and reared in individual pens with artificial milk powder fed to them in buckets until $6 \mathrm{wk}$ of age, thereafter being reared in groups fed conserved forage and concentrates until calving at $2 \mathrm{yr}$ of age. The dairy cows were divided into three groups: high yielders, low yielders, and nonlactating, with each cow experiencing at least two changes of group per lactation. Mean annual milk yield was $7500 \mathrm{~L} / \mathrm{cow}$. From August to May the cows are fed a TMR of grass silage and concentrates, in a covered straw yard at a stocking density of $12 \mathrm{~m}^{2}$ per cow. From May to August, the cows grazed perennial ryegrass pasture (Lolium perenne), with access to a food supplement after milking. During the study, the cows were grazed day and night at a stocking rate of 5 cows/ha, and received a supplement of wheat feed for $30 \mathrm{~min}$ following the afternoon milking before returning to pasture. Cows were inseminated artificially and were milked by machine in a rapid exit parlor (Alfa-Laval, Camberley, Surry) twice daily at 0600 and $1500 \mathrm{~h}$.

The frequency of behavior recording was less than in experiment 1 because 142 milking cows were recorded. It also differed between behaviors according to ease of 
collecting data. Lying (recorded as in experiment 1, except that the cows were at pasture), feeding (tongue protrusion from one side of the mouth during the initiation of a feeding bout while eating the supplement), rumination (jaw movement while ruminating, recorded during milking), and track (the side of the track leading from the farm buildings to their field, which they selected after consuming their supplement in the afternoon) were observed three times for each cow. The track, which was formed from two lines of electric fencing across a field, was of length $100 \mathrm{~m}$ and width $5 \mathrm{~m}$ and contained two well-worn routes on either side. For most behaviors, this recording schedule resulted in approximately the same total number of observations, 426 (142 cows $\times 3$ observations) per behavior, as in the first experiment, which had 440 (40 cows $\times 11$ observations) per behavior. The side of the parlor that cows voluntarily selected was recorded seven times (parlor) due to the ease of recording. Tail waving was not regularly observed and was therefore not included in the behaviors recorded. Observations were made by a single individual close to the herd between 1330 and $1430 \mathrm{~h}$ daily during the month of July.

Statistical analysis. All behavior variables were tested for normality of data distribution by the Kolmogorov-Smirnov and Anderson-Darling tests using the statistical package Minitab. No behaviors conformed to a normal distribution, nor could the data for any one be transformed mathematically to meet the requirements of a normal distribution. Therefore, for each behavior, cows were classified as consistent (behavior always demonstrated on the same side) or nonconsistent (behavior exhibited on more than one side). In addition, behaviors were awarded a score of 0 if the cow exhibited the behavior on the left side and 1 if the cow exhibited the behavior on the right side, as in experiment 1 , and a mean score for each cow determined for each behavior. The correlations of laterality between the mean scores for each cow were analyzed by Spearman-rank correlation coefficients using Minitab (1995). A contingency table was constructed to investigate the association between behaviors for consistent cows.

\section{RESULTS}

\section{Experiment 1}

The normal distribution of the data for all behaviors by the Kolmogorov-Smirnov test indicated a predominance of cows with low laterality (Figure 1). There were significant positive Spearman-rank correlations between laterality exhibited during rumination and Lying, rumination and walking, walking and tail, and walking and feeding (Table 1). A stepwise regression between the laterality exhibited during walking and during other behaviors found that all three correlated variables were significant at $P<0.10$ (Equation 1), indicating that there was a tendency for these variables to be directly related to walking.

$$
\begin{aligned}
\text { Walking }= & 0.32( \pm 0.13, P=0.02) \text { rumination }+0.52 \\
& ( \pm 0.17, P=0.003) \text { tail }+0.22 \\
& ( \pm 0.12, P=0.09) \text { feeding. }
\end{aligned}
$$

There was no evidence of nonlinear relationships between variables.

\section{Experiment 2}

Following the evidence that behavioral data was not normally distributed for any behavior (Figure 2), the mean scores for laterality were calculated as $0.50,0.54$, $0.46,0.55$, and 0.54 for parlor, rumination, feeding, lying, and track, respectively. This did not differ significantly from 0.5 for any behavior when examined by the chi-square test, indicating that neither the right nor left sides were dominant.

For each behavior, two populations of cows emerged within the herd, showing either consistent (all recordings on one side) or inconsistent (recordings on both sides) laterality (Figure 2). The proportion of cows demonstrating consistent laterality varied significantly $(P$ $<0.05$ ) between the behaviors, when tested by a contingency table. According to binomial probability theorem, under random distribution $25 \%$ of cows should be consistent for behaviors recorded three times, and fewer than $1 \%$ should be consistent for parlor entry behavior, which was recorded seven times. When observed and expected proportions of consistent cows were tested by chi-square, the proportion of consistent cows was greater than expected for feeding $(88 \%, P<0.001)$, lying (51\%, $P<0.001)$, parlor $(43 \%, P<0.001)$, and rumination $(37 \%, P<0.01)$, but not for track $(56 \%, P>0.10)$. After excluding the consistent cows, the distribution of the remaining cows was still not normal for any behavior, by the Anderson Darling test (Minitab, 1995).

As the data were not normally distributed, associations were tested by ranking the data and calculating one-tailed Spearman-rank correlation coefficients (Minitab, 1995). There were no significant correlations $(P>0.10)$ between the ranked laterality scores of any two behaviors, except that parlor and track were positively correlated ( $\mathrm{t}=0.162, P=0.06)$. Therefore cows that went to one side of the parlor for milking also tended to exit the farm to pasture on the same side of the farm track.

\section{DISCUSSION}

The total number of recordings per experiment was estimated in the absence of knowledge of the number 

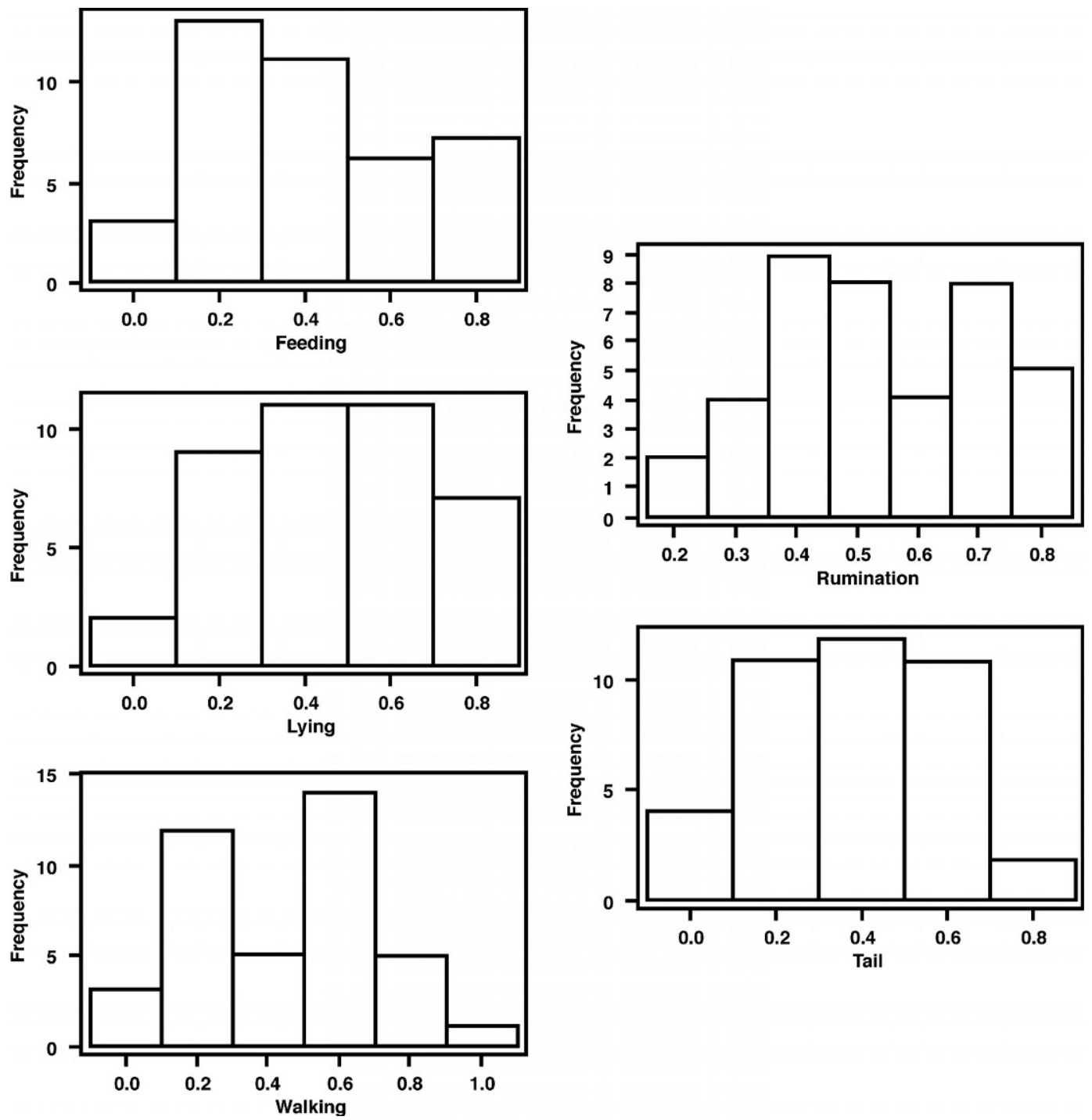

Figure 1. Frequency distribution of cows exhibiting left or right side laterality $(0=$ consistent left side laterality, $1=$ consistent right side laterality) for feeding, lying, walking, rumination, and tail behaviors in Experiment 1.

Table 1. Spearman rank correlation coefficients for rumination, tail, feeding, lying, and walking behaviors in experiment $1 .^{1}$

\begin{tabular}{lclll}
\hline & Rumination & Tail & Feeding & Lying \\
\hline Tail & 0.138 & & & \\
Feeding & -0.031 & 0.079 & & \\
Lying & $0.318^{*}$ & 0.076 & 0.006 & \\
Walking & $0.335^{*}$ & $0.492^{* *}$ & $0.287 \dagger$ & 0.161 \\
\hline
\end{tabular}

${ }^{1}$ Rumination $=$ dominant side of the mouth occupied by the lower jaw during ruminating; tail = which side of the body the tail was initially moved to during tail swishing; feeding = tongue protrusion from one side of the mouth during the initiation of a grazing bout; lying = which hind leg was under the body during lying; walking = which front leg they moved first after standing still.

$$
\begin{aligned}
& \dagger P=0.06 . \\
& * P<0.05 . \\
& * * P<0.01 .
\end{aligned}
$$

of recordings needed to demonstrated normality (or otherwise). However, the results from experiment 2 suggest that the data for some behaviors could be proven to lack normal distributions with just three observations per animal.

The normal distribution of laterality in cows in the extensively managed herd agrees with studies of other animals reared naturally. Extreme laterality can be caused by circumstances in which animals or, in particular, humans are required, or elect, to perform tasks repeatedly on one side. It can also be caused by exposure to stress in primates at least, as evidenced by high plasma cortisol concentrations (e.g., Westergaard et al., $2000,2001)$. It is possible that the cows in the intensively managed herd were subjected to greater stress, 

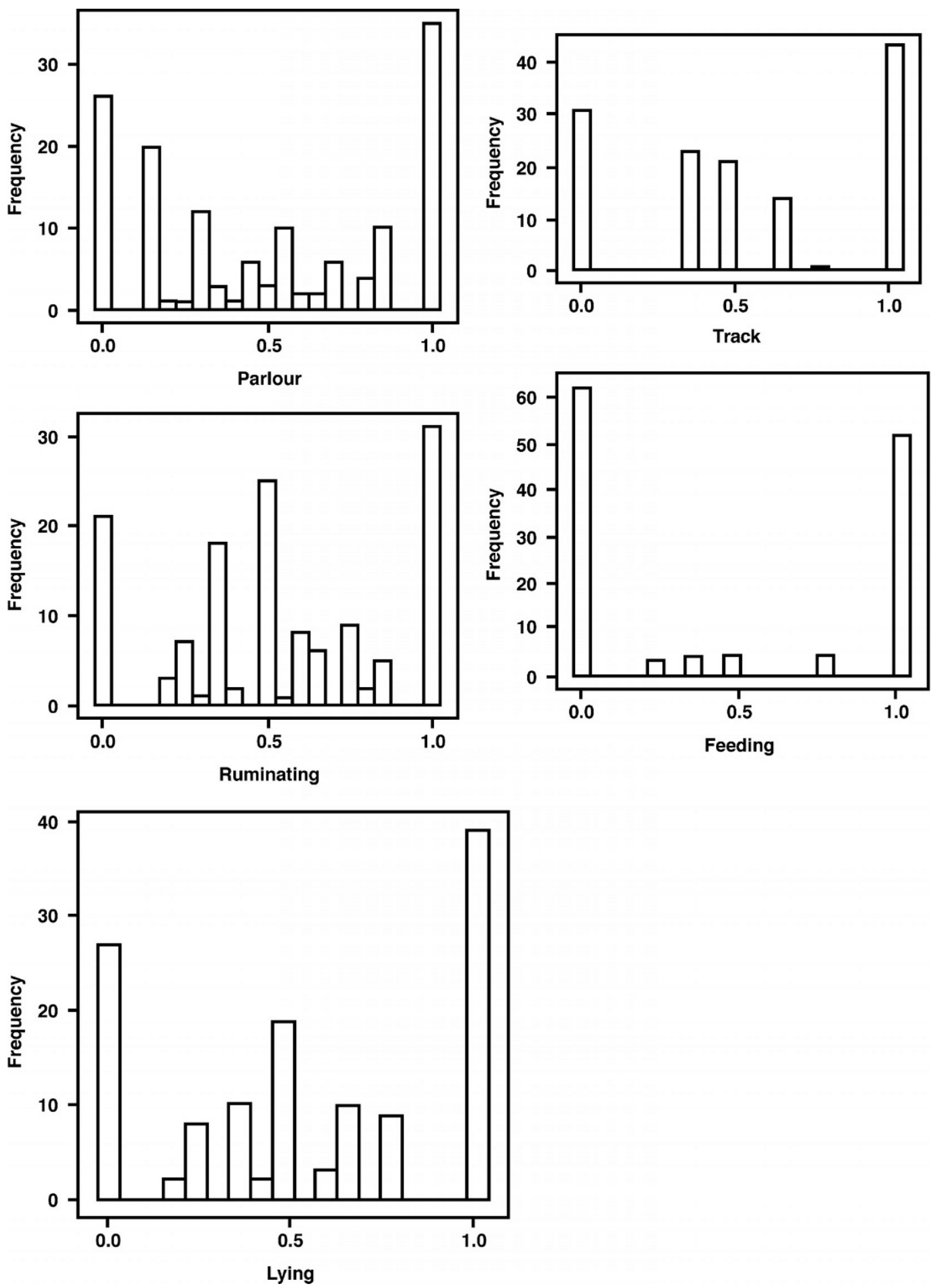

Figure 2. Frequency distribution of cows exhibiting left or right side laterality $(0=$ consistent left side laterality, $1=$ consistent right side laterality) for parlor entry, rumination, track, feeding, and lying behaviors in experiment 2.

although we obtained no direct evidence of this. However, it is conceivable that they were exposed to greater social stresses during rearing, because they were sepa- rated at $1 \mathrm{~d}$ of age from their mothers and reared in individual pens. The early separation in the British herd is not a natural herd behavior (Reinhardt and 
Reinhardt, 1981) and is associated with a brief increase in heart rate and vocalization (Hopster et al., 1995). Cortisol has increased associated with weaning or separation from several weeks to a few months of age in beef cattle studies (Whisnant et al., 1985; Lefcourt and Elsasser, 1995) but not for separation shortly after birth in dairy cattle (Hopster et al., 1995). Epinephrine and norepinephrine may be better hormonal indicators of distress. In calves separated from their dams at 4 to 6 mo of age, epinephrine increases for at least $1 \mathrm{~d}$ following weaning (Lefcourt and Elasser, 1995), indicating that the situation of the calves may be uncontrollable, whereas norepinephrine increased in their dams, suggesting better control, perhaps due to repeated exposure to the stressor. However, there likely are differences in the degree of stress with separation shortly after birth in dairy cattle compared with separation after a cow-calf bond is established, more typical of beef production systems. In addition to early weaning, individual rearing used for the British, but not the Brazilian calves may be associated with abnormal behavior (Margerison et al., 2002). The British cows were kept inside for most of the year at a high stocking density, compared with the Brazilian cows that were kept outside, their preferred environment (Krohn et al., 1992). The British cows were also moved regularly between groups, which increases aggression and reduces milk yield during the first few weeks after moving to a new group (Phillips and Rind, 2002). Laterality in parlor side selection has been demonstrated previously (Hopster et al., 1998; Paranhos da Costa and Broom, 2001), and consistent side selection correlates with dominance (Prelle et al., 2001). Hopster et al. (1998) found a high level of consistency in cow selection of parlor side in a similar management system to that used for the British herd in this study. In a large group of laying hens, fluctuating asymmetry is positively correlated with a low stress-buffering capacity, and both cannibals and victims are more asymmetrical than other birds (Yngvesson and Keeling, 2001). Dominant cows may be stressed by their need to maintain their position in the dominance order (Phillips and Rind, 2001), and dominance orders form more readily in cattle reared in isolation than those reared in groups (Broom and Leaver, 1978). Dominance is most likely to have an influence on the consistency of laterality if there is competition between cows. Given the limited interaction between cows in the other behaviors where cattle displayed laterality, compared with parlor entry, it is unlikely that dominance was a major factor for most of the behaviors recorded, unless related to stress. In free stalls, factors such as the presence of another cow in a neighboring cubicle may influence laterality (Hayasaka et al., 1992). The extreme laterality shown by the British cows may reflect a strategy of consistent behavior, which minimizes the need for decision-making in unimportant issues, reserving capacity for more important decisions. For example, entering the "abnormal" side of the milking parlor causes only minor stress, as evidenced by effects on heart rate (Hopster et al., 1998), which could be avoided by a consistent approach to parlor entry. Extreme laterality may also relate to social structure, and it can be hypothesized that the British herd had a well-formed hierarchy, with consistent behavior helping to diminish the scale of agonistic interactions. Inconsistent behavior in the Brazilian cows may reflect an ability to adapt to circumstances, a coping strategy for life in a variable environment. Other possible influences on laterality include differences in genetic inheritance. The two herds contained cows of the same breed, but the greater milk production of the British cows was probably partly due to increased genetic potential for milk production compared with the Brazilian cows. An influence of genetic inheritance is well established for handedness in humans, but not for lateral tendencies for eye, ear, hand, and foot movements (Saudino and McManus, 1998; Reiss et al., 1999). The herds were also shown to be different in the degree of correlation between laterality in different behaviors. The absence of laterality correlations in the British herd, except in the similar behaviors of parlor-side selection and track side selection, suggests that the lateralities derived from disparate forces. This may relate to early experience; for example, the side of their rearing pen on which the food was presented may have influenced the side of the mouth from which the tongue was protruded. In contrast, the correlations between lateralities in the Brazilian herd were stronger, which may reflect inheritance or patterns of behavior learned from their mothers, a facility scarcely afforded to calves in the British herd. Future research could explore interherd differences, in particular the influence of herd management systems and genetics. Only two herds were examined in the current study, and the many differences between herds confound interpretation of observed differences in laterality of various behaviors. Because asymmetry in limb use takes two forms-differential limb preference and differential dexterity between the limbs (Reiss and Reiss, 1998) - it is important to establish whether cows are ambidextrous.

\section{CONCLUSIONS}

There were differences between herds in the extent of behavioral laterality. In an extensively managed herd there was a normal distribution of laterality for behaviors performed predominantly or partially on one side of the body. The extent of laterality was correlated be- 
tween behaviors, indicating a possible common etiology. In contrast, cows in an intensively managed herd consistently showed these behaviors on one side of the body, and there was little correlation between the behaviors. Because a relationship between laterality and stress has been observed in other species, possibly higher stress levels in the intensively managed herd may be a factor. However, influence of other factors such as dominance, genetics, and experience, cannot be discounted.

\section{ACKNOWLEDGMENTS}

The authors are grateful to Jose Manoel Teixera of Fazenda Campoleina, Brazil, and Eric Allen of the Cambridge University Farm for permission to observe the cows.

\section{REFERENCES}

Albright, J. L., and C. W. Arave. 1997. The Behavior of Cattle. CAB International, Wallingford, UK.

Annett, M. 1985. Left, Right, Hand and Brain: The Right Shift Theory. A. Wheaton \& Co. Ltd., Exeter, UK.

Arave, C. W., and J. L. Walters. 1980. Factors affecting lying behavior and stall utilization of dairy cattle. Appl. Anim. Ethol. 6:369-376.

Arave, C. W., R. C. Lamb, M. J. Arambel, D. Purcell, and J. L. Walters. 1992. Behavior and maze learning ability of dairy calves as influenced by housing, sex and sire. Appl. Anim. Behav. Sci. 33:149-163.

Bao, J., and P. S. Giller. 1991. Observations on the changes in behavioral activities of dairy cows prior to and after parturition. Irish Vet. J. 44:43-47.

Bard, K. A., W. D. Hopkins, and C. L. Fort. 1990. Lateral bias in infant chimpanzees (Pan troglodytes). J. Comp. Psychol. 104:309-321.

Broom, D. M., and J. D. Leaver. 1978. The effects of group-housing or partial isolation on later social behavior of calves. Anim. Behav. 26:1255-1263.

Davis, P. J. 1988. Physiological and subjective effects of catharsis: A case report. Cognit. Emotion 2:19-28.

Ewbank, R. 1992. Stress: A general overview. Pages 255-262 in Farm Animals and the Environment. C. J. C. Phillips and D. Piggins, ed. CAB International, Wallingford, UK.

Gangestad, S. W., and R. A. Yeo. 1994. Parental handedness and relative hand skill: A test of the developmental instability hypothesis. Neuropsychology 8:572-578.

Hayasaka, K., N. Yamagishi, N. Takusari, and T. Miyauchi. 1992. Factors affecting laterality of lying down and changes to the stallfed Holstein cows. Jpn. J. Livest. Manage. 28:43-49.

Hopster, H. J., T. N. van der Werf, and H. J. Blokhuis. 1998. Side preference in dairy cows in the milking parlor and its effects on behavior and heart rate during milking. Appl. Anim. Behav. Sci. 55:213-229.

Hopster, H., J. M. O'Connell, and H. J. Blokhuis. 1995. Acute effects of cow-calf separation on heart rate, plasma cortisol and behavior in multiparous dairy cows. Appl. Anim. Behav. Sci. 44:1-8.
Krohn, C. C., L. Munksgaard, and B. Jonasen. 1992. Behavior of dairy cows kept in extensive (loose housing pasture) or intensive (tie stall) environments 1. Experimental procedure, facilities, time budgets-diurnal and seasonal conditions. Appl. Anim. Behav. Sci. 34:37-47.

Lefcourt, A. M., and T. H. Elsasser. 1995. Adrenal responses of Angus $\times$ Hereford cattle to the stress of weaning. J. Anim. Sci. 73:2669-2676.

Margerison, J. K., T. R. Preston, N. Berry, and C. J. C. Phillips 2002. Cross sucking and other oral behaviours in calves and their relation to cow suckling and food provision. Appl. Anim. Behav. Sci. 80:277-286.

Minitab. 1995. Minitab Reference Manual, Release 10Xtra for Windows and Macintosh. Minitab Inc., State College, PA.

Paranhos da Costa, M. J. R., and D. M. Broom. 2001. Consistency of side choice in the milking parlor by Holstein-Friesian cows and its relationship with their reactivity and milk yield. Appl. Anim. Behav. Sci. 70:177-181.

Phillips, C. J. C., and M. I. Rind. 2001. The effects on production and behavior of mixing uniparous and multiparous cows. J. Dairy Sci. 84:2424-2429.

Phillips, C. J. C., and M. I. Rind. 2002. The effects of social dominance on the production and behavior of grazing dairy cows offered forage supplements. J. Dairy Sci. 85:51-59.

Phillips, C. J. C., S. J. Patterson, I. Ap Dewi, and C. J. Whittaker 1996. Volume assessment of the bovine hoof. Res. Vet. Sci. 61:125-128.

Prelle, I., C. J. C. Phillips, and D. M. Broom. 2001. Does consistent choice of one side of a milking parlor by dairy cows relate to their behavior in novel and competitive situations? PC 11 in Proc. Int. Soc. Appl. Ethol. Regional Mtng., York, UK.

Reinhardt, V., and A. Reinhardt. 1981. Cohesive relationships in a cattle herd (Bos indicus). Behavior 77:121-151.

Reiss, M., and G. Reiss. 1998. Einige Aspekte der Lateralitatsuntersuchung. (Certain aspects of laterality research). Arch. Kriminol. 201:103-111.

Reiss, M., G. Tymnik, P. Koegler, W. Koegler, and G. Reiss. 1999. Laterality of hand, foot, eye, and ear in twins. Laterality 4:287-297.

Rodriguez-Diaz, D., and D. Afonso. 1993. Ontogeny of T-maze behavioral lateralization in rats. Phys. Behav. 54:91-94.

Saudino, K., and I. C. McManus. 1998. Handedness, footedness, eyedness and earedness in the Colorado Adoption Project. Br. J. Dev. Psychol. 16:167-174.

Tang, A., and T. Verstyned. 2002. Early life environment modulates 'handedness' in rats. Behav. Brain Res. 131:1-8.

Uhrbrook, R. S. 1969. Bovine Laterality. J. Gen. Psychol. 115:77-79.

Westergaard, G. C., T. J. Chavanne, I. D. Lussier, S. J. Suomi, and J. D. Higley. 2000. Hormonal correlates of hand preference in free-ranging primates. Neuropsychopharmacology 23:502-507.

Westergaard, G. C., L. D. Lussier, S. J. Suomi, and J. D. Higley. 2001. Stress correlates of hand preference in rhesus macaques. Dev. Psychobiol. 38:110-115.

Whisnant, C. S., T. E. Kiser, and F. N. Thompson. 1985. Effect of calf removal on serum luteinizing hormone and cortisol concentrations in beef cows. Theriogenology 24:119-125.

Wilson, L., L. Terosky, C. L. Stull, and W. R. Stricklin. 1999. Effects of individual housing design and size on behavior and stress indicators of special-fed Holstein veal calves. J. Anim. Sci. 77:1341-1347.

Yngvesson, J., and L. J. Keeling. 2001. Body size and fluctuating asymmetry in relation to cannibalistic behavior in laying hens. Anim. Behav. 61:609-615. 